\title{
The suppression of hydrodynamic noise from underwater cavities by the change of back wall chamfer
}

\author{
Hao Zhang ${ }^{1,2,3}$, Yongwei Liu ${ }^{1,2,3, *}$, and Dejiang Shang ${ }^{1,2,3}$ \\ 1. Acoustic Science and Technology Laboratory, Harbin Engineering University, Harbin 150001, China \\ 2. Key Laboratory of Marine Information Acquisition and Security (Harbin Engineering University), Ministry of Industry and \\ Information Technology; Harbin 150001, China \\ 3. College of Underwater Acoustic Engineering, Harbin Engineering University, Harbin 150001, China
}

\begin{abstract}
When underwater vehicles are sailing, high hydrodynamic noise will be generated through the opening cavities due to the interaction of the surface and the fluids. In the paper, we had tested different forms of back wall chamfer by numerical calculation based on the method of large eddy simulation, to control the impact of the eddy and break the flow at the trailing edge of the cavity, which is with the dimension of $100 \mathrm{~mm}$ wide, $120 \mathrm{~mm}$ high and $100 \mathrm{~mm}$ long. The angle and shape of the trailing edge chamfer are changed to control the flow of the cavity. We had also investigated the effect of hydrodynamic noise suppression through the simulation, which is based on the method of Lighthill's acoustic analogy. The results show that the change of back wall chamfer can stabilize the movement of the eddies inside the cavity and reduce the fluctuation pressure at the trailing edge of the cavity. The suppression of flow-induced noise can be up to $5 \mathrm{~dB}$, if the back-wall chamfer is with the airfoil surface and the angle of back wall chamfer is properly designed.
\end{abstract}

\section{Introduction}

The radiated noise of underwater vehicle is mainly composed of the mechanical noise, propeller noise and hydrodynamic noise. With the increase of the speed, hydrodynamic noise has gradually become an important part in underwater sound radiation. Although all underwater vehicles are streamlined, there inevitably are various flow holes on the surface. The water holes form the structure of underwater cavity. The surface of the cavity will form turbulence, and the unstable flow will radiate noise. At the same time, the fluctuation pressure produced by the fluid can also excite the cavity to generate structural vibration, and the wall radiates noise because of the coupling with water. The hydrodynamic noise of the cavity seriously destroys the acoustic stealth performance of the submarines.

The oscillation of the cavity shear layer was first studied by Rayleigh. He believed that the shear layer oscillation can be understood as the result of fluid conservation of the internal angular momentum. Rossiter proposed a semi-empirical and semi-theoretical formula to calculate the frequency of cavity oscillation in transonic and supersonic flow[1]. Rockwell experimenttally proposed that the interaction between the shear layer and the fluid outside the cavity is the source of cavity oscillation[2].

Seiichiro IZAWA used a thin plate inserted into the cavity as a passive flow control device and found that the plate achieved the noise suppression up to $14 \mathrm{~dB}[3]$. Brenda Henderson shows that the water jet reduces the total sound pressure level by about $6 \mathrm{~dB}$, and the gas jet can reduce the total sound pressure level by about 2 $\mathrm{dB}[4]$. N. Heeb's experiments have shown that hydrodynamic noise can be reduced by both jet injection and serrated construction[5]. Yiping Wang found that the upstream concave surface can effectively suppress the cavity flow oscillation[6]. The flow control is a commonly method, which is divided into two categories: passive and active control. Among them, the passive flow control technology has the advantages of simple structure and convenient application. For aerodynamic noise, the method of changing the back-wall chamfer has been adopted to reduce noise[7]. However, the application of back wall chamfer in underwater cavities is still insufficient, and the relationship between the chamfer and hydrodynamic noise is still unknown.

\section{Principle and method verification of simulation}

\subsection{Numerical calculation method of fluid dynamics}

The turbulence simulation method can be roughly divided into direct numerical simulation and indirect numerical simulation. Direct numerical simulation directly solves the turbulent N-S equation to obtain all

\footnotetext{
${ }^{*}$ Corresponding author: liuyongwei3000@hrbeu.edu.cn
} 
the information of the instantaneous flow field. This method is computationally intensive and cannot be used to solve complex flow problems. Therefore, people introduce some kind of averaging concept to reduce the amount of calculation, so that the fundamental equations of fluid mechanics that are originally closed have new unknowns. It is necessary to introduce a series of assumptions to the equations to close them. This kind of assumption is called turbulence model. In this paper, the numerical simulation is carried out by the method of large eddy simulation.

The Navier-Stokes equation (N-S equation) is the basic governing equation for describing fluid motion. The water is incompressible fluid, so the flow state obeys the N-S equation. The N-S equation is described in the Cartesian coordinate system as follows

$$
\begin{aligned}
\frac{\partial \mu_{i}}{\partial t}+\frac{\partial\left(\mu_{i} \mu_{j}\right)}{\partial x_{j}} & =f_{i}-\frac{1}{\rho} \frac{\partial p}{\partial x_{i}}+v \frac{\partial^{2} \mu_{i}}{\partial x_{i} \partial x_{j}} \\
\frac{\partial \mu_{i}}{\partial x_{i}} & =0
\end{aligned}
$$

where $\rho$ represents fluid density; $v$ represents a moving viscosity coefficient; $f_{i}$ is a mass force intensity; $u_{i}$ represents a fluid velocity; and $p$ is a fluid pressure.

The basic idea of large eddy simulation (LES) is to first establish a filter function, substituting the parameters of the large-scale vortex into the instantaneous N-S equation, and then processing the small-scale vortex filtered out as a sub-grid model. The effect of large-scale vortices is reflected by adding a sub-grid stress term (SGS) to the equation of flow field motion. The N-S equation filtered by the filter function is as follows

$$
\begin{gathered}
\frac{\partial}{\partial t}\left(\rho \bar{u}_{i}\right)+\frac{\partial}{\partial x_{i}}\left(\rho \bar{u}_{i} \bar{u}_{j}\right)=-\frac{\partial \bar{p}}{\partial x_{i}}+\frac{\partial}{\partial x_{j}}\left(\mu \frac{\partial \bar{u}_{i}}{\partial x_{i}}\right)-\frac{\partial \tau_{i j}}{\partial x_{i}} \\
\tau_{i j}=\rho \overline{u_{i} u_{j}}-\rho \bar{u}_{i} \bar{u}_{j}
\end{gathered}
$$

In the formula, is a sub-grid stress (SGS), which is an unknown quantity, so a closed model of sub-grid stress is constructed. This paper uses the Dynamic sub-grid model in the form

$$
\tau_{i j}-\frac{1}{3} \tau_{k k} \delta_{i j}=-2 \mu_{t} \bar{S}_{i j}
$$

In the formula, $\mu_{t}=\left(C_{s} \Delta\right)^{2}|\bar{S}|$ is the sub-grid eddy viscosity coefficient.

\subsection{Lighthill acoustic analogy theory}

The Lighthill acoustic analogy theory combines the flow field information with the sound field information by transforming the N-S equation to solve the problem of how to establish the sound source in the flow noise.

The continuity equation of the turbulent flow field is as follows

$$
\frac{\partial \rho}{\partial t}+\frac{\partial \rho v_{i}}{\partial x_{i}}=0
$$

Obtained from the turbulent flow field N-S equation

$$
\frac{\partial \rho v_{i}}{\partial t}+\frac{\partial \rho v_{i} v_{j}}{\partial x_{j}}=-\frac{\partial P_{i j}}{\partial x_{j}}
$$

here, $P_{i j}=P \delta_{i j}-\tau_{i j}, \tau_{i j}$ is a viscous stress.

$$
\text { Add } c_{0}^{2} \frac{\partial \rho}{\partial x_{i}} \text { to each side of the above formula }
$$

(where $c_{0}$ is the speed of sound), and the above formula can be rewritten as

$$
\frac{\partial \rho v_{i}}{\partial t}+c_{0}^{2} \frac{\partial \rho}{\partial x_{i}}=-\frac{\partial T_{i j}}{\partial x_{i}}
$$

where the stress tensor $\mathrm{T}$ is

$$
T_{i j}=\rho v_{i} v_{j}+\delta_{i j}\left[\left(P-P_{0}\right)-c_{0}\left(\rho-\rho_{0}\right)\right]-\tau_{i j}
$$

For ideal fluids

$$
\frac{\partial^{2}}{\partial t^{2}}\left(\rho-\rho_{0}\right)-c_{0}{ }^{2} \frac{\partial^{2}}{\partial x_{i} \partial x_{j}}\left(\rho-\rho_{0}\right)=\frac{\partial^{2} T_{i j}}{\partial x_{i} \partial x_{j}}
$$

where $\rho_{0}$ is the environmental density, $P_{0}$ is the ambient sound pressure, $\rho_{a}, P_{a}$ is the acoustic variable.

$$
\begin{aligned}
& \text { Let } \rho_{a}=\rho-\rho_{0}, P_{a}=P-P_{0}, \text { then } \\
& \frac{\partial^{2} \rho_{a}}{\partial t^{2}}-c_{0}{ }^{2} \frac{\partial^{2} \rho_{a}}{\partial x_{i} \partial x_{j}}=\frac{\partial^{2} T_{i j}}{\partial x_{i} \partial x_{j}} \\
& T_{i j}=\rho v_{i} v_{j}+\delta_{i j}\left[\left(P-P_{0}\right)-c_{0}^{2}\left(\rho-\rho_{0}\right)\right]-\tau_{i j}
\end{aligned}
$$

The viscous stress $\tau_{i j}$ is a negligible small amount relative to the Reynolds stress; and $\frac{\rho_{a}}{\rho} \ll 1, \frac{P_{a}}{\rho} \ll 1$, So we have equation (12)

$$
\begin{aligned}
& \frac{\partial^{2} \rho_{a}}{\partial t^{2}}-c_{0}^{2} \frac{\partial^{2} \rho_{a}}{\partial x_{i} \partial x_{j}}=\frac{\partial^{2} T_{i j}}{\partial x_{i} \partial x_{j}} \\
& T_{i j}=\rho v_{i} v_{j}+\delta_{i j}\left[\left(P-P_{0}\right)-c_{0}\left(\rho-\rho_{0}\right)\right]-\tau_{i j}
\end{aligned}
$$

The above formula is the basic equation for turbulent fluid vocalization.

\subsection{Numerical simulation method verification}

The flow-induced noise simulation was verified using a simple support plate.

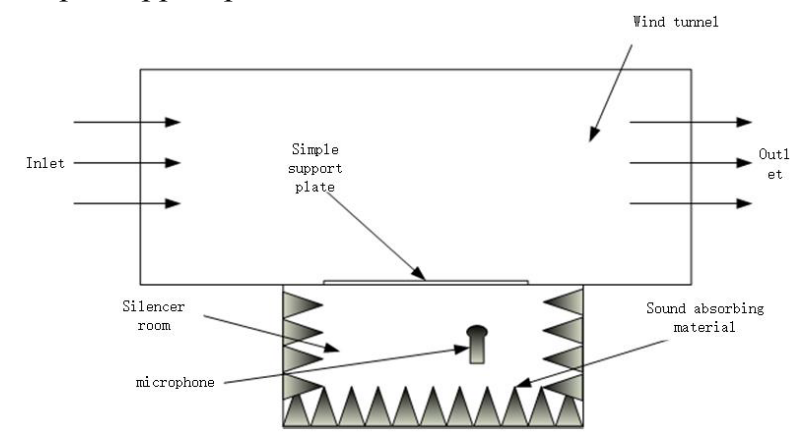

Fig. 1. Simple support plate wind tunnel test and measurement device. 
Heatwole C.M [8,9]and others have carried out simulation calculation and experimental research on the flow noise of the simple support plate. The test device is shown in the figure, the simple support plate is embedded on the wall surface of the wind tunnel, and the rear end of the simple support plate is a small muffler chamber. A microphone is installed to measure the radiated noise sound pressure of the simple support. The material and size parameters of the simple support plate are as follows: material, aluminum; length $0.46 \mathrm{~m}$; width $0.33 \mathrm{~m}$; thickness $0.0048 \mathrm{~m}$; density $2.7 * 10^{3} \mathrm{~kg} / \mathrm{m}^{3}$; Young's modulus $7.3 * 10^{10} \mathrm{~N} / \mathrm{m}^{2}$; Poisson's ratio 0.33 . Taking the top angle of the board as the origin, the microphone is located at the coordinates $(0.458 \mathrm{~m}$, $-0.035 \mathrm{~m}$, and $0.458 \mathrm{~m})$. The flow-induced radiation noise of the simple support plates at three flow rates of $26.8 \mathrm{~m} / \mathrm{s}, 35.8 \mathrm{~m} / \mathrm{s}$ and $40.2 \mathrm{~m} / \mathrm{s}$ is measured.

In this paper, the flow noise of the above simple support plate is numerically calculated. The boundary conditions used in the calculation are the same as those provided by Heatwole C.M, and the flow rate is calculated to be $35.8 \mathrm{~m} / \mathrm{s}$.

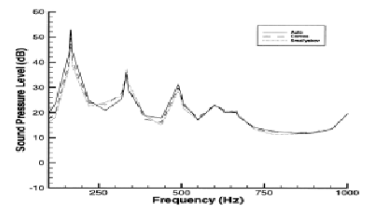

Results in the literature

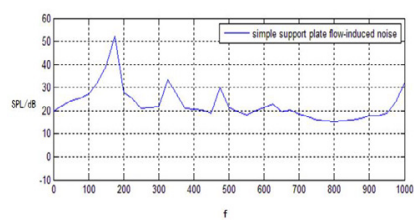

Simulation results
Fig. 2. Comparison of results.

The simulation verifies the accuracy of the numerical calculation method for the flow noise of the plate.

\section{Modelling and simulation}

There are two main parts of the research. Firstly, we had changed the angle of the back wall of underwater cavity, and analyze how the chamfer of each angle changes the flow-induced noise of the cavity by the change of flow field. Secondly, on the basis, the air foil surface is used to change the plane chamfer, and the influence of the air foil surface chamfer on the flow-induced noise of underwater cavity is summarized.

\subsection{Flow field}

We had created a three-dimensional cavity model without chamfer. For the submarine, the shape of the opening of the drain hole is mostly rectangular. For an actual underwater opening cavity, the boundary conditions and the parts that are not of interest can be simplified. We had set up the following models. The cavity is in hexahedron of $100 \mathrm{~mm}$ long, $100 \mathrm{~mm}$ wide and $150 \mathrm{~mm}$ high. An external flow field of the cavity is established at the opening of the cavity. The distance between the cavity opening and the inlet of flow field, the outlet of flow field, the left boundaries, and the right boundaries is $100 \mathrm{~mm}, 200 \mathrm{~mm}, 100 \mathrm{~mm}, 100 \mathrm{~mm}$, respectively.

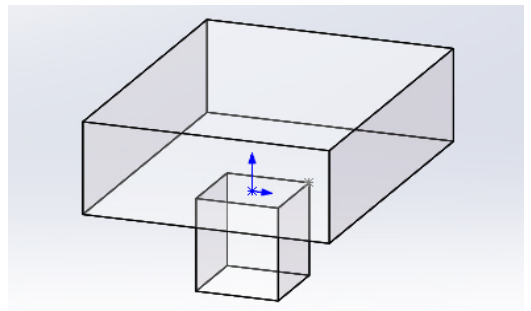

Fig. 3. The original model of flow field.

The chamfer is added on the original model. The angle of the back-wall chamfer is $30^{\circ}, 45^{\circ}, 60^{\circ}$, respectively. After the radiation noise is calculated, the air foil surface is added to the back-wall chamfer with better effect of noise reduction. The bottom surface of cavity wall and the outer flow field model are set up with no slip wall surface. The flow field inlet is set as the velocity inlet $(10 \mathrm{~m} / \mathrm{s})$, the flow field outlet is set as the pressure outlet, and the other three sides of outer flow field are set as the symmetry.

chamfer with

different
angles
chamfer with
different
shapes
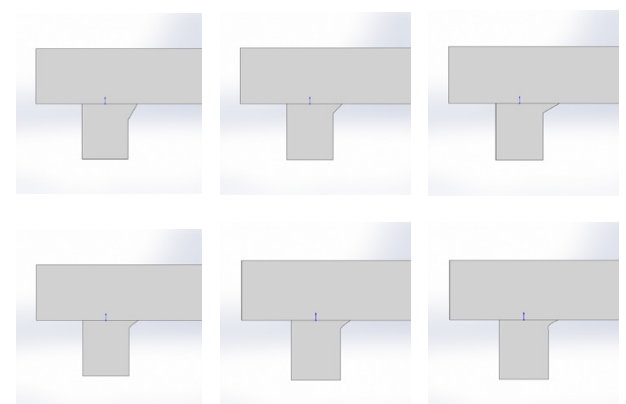

Fig. 4. Model with back wall chamfer.

The sound field is solved after the data of flow field is calculated by the large eddy simulation. Since the large eddy simulation is sensitive to the initial flow field conditions, the better initial conditions can greatly reduce the iteration time, compared to the simple initialization conditions, and can reduce the possibility of numerical divergence. Therefore, it is necessary to perform steady state calculation on the flow field, and the convergence result is used as the initial flow field condition of the large eddy simulation. The steady state calculation uses the standard k- $\omega$ model. For transient calculations, the subgrid-scale model is the Dynamic Smagorinsk-Lilly model. The pressure-velocity coupling algorithm is SIMPLE algorithm. And we chose $0.00025 \mathrm{~s}$ as the calculation time step, if the analysis frequency is $2000 \mathrm{~Hz}$.

\subsection{Sound field}

For the underwater vehicle, the Mach number is extremely low. At the time, the flow-induced noise in the hydrodynamic noise of the cavity is dominant, much larger than the flow noise, and the contribution of flow noise is very little to the sound field. Only the flow-induced noise can be considered in the simulation of sound field. The model of sound field is designed as a sphere with the radius of $260 \mathrm{~mm}$. The surface of the sphere is constructed by the infinite element method to simulate the free field. The inner sound field material is water. The three-dimensional cavity is set up as 
thin-shell element, the material is defined as steel, and the thickness is $5 \mathrm{~mm}$.

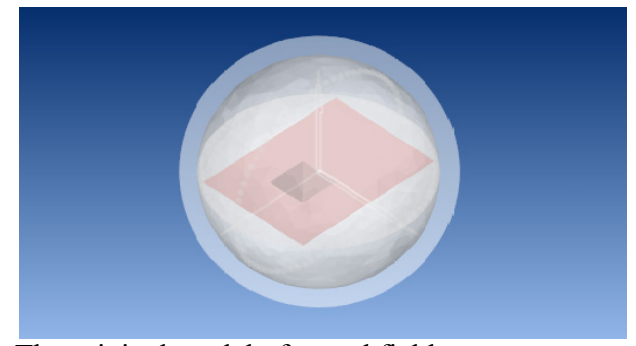

Fig. 5. The original model of sound field.

To mesh the sound propagation domain and completely describe the information of sound wave, it is necessary to ensure that 6-8 nodes per wavelength are used in the first-order unit, and 4 nodes per wavelength are required in the second-order unit. We choose the minimum wavelength as the standard for the definition of grid size with high accuracy. In the software of ACTRAN, 1/10 of the minimum wavelength is generally considered as the maximum grid scale to obtain more accurate results.

The results of flow field calculation, the flow field calculation grid information, and the sound calculation grid information are imported into the ICFD module of ACTRAN, and then the calculation result is interpolated and transformed by the Fourier. The fluctuation pressure on the surface of underwater cavity is extracted and converted into the excitation force, which acts on the surface of the cavity, causes the vibration of the wall surface of the cavity to radiate noise.

\section{Influence of back wall chamfer on hydrodynamic noise of cavity}

\subsection{Influence of chamfer angle on hydrodynamic noise of cavity}

The sound field of three different angle models is compared with that of the original model. The sound pressure level of back wall chamfer at the angle of $45^{\circ}$ has the rapid oscillation change with the frequency. A possible reason is that the characteristic frequency of the solution is not unique in the calculation of boundary element, which is easily to result in the periodic oscillations with the frequency. The surface in our study is the NACA airfoil surface, which is upper airfoil and widely used in the water.

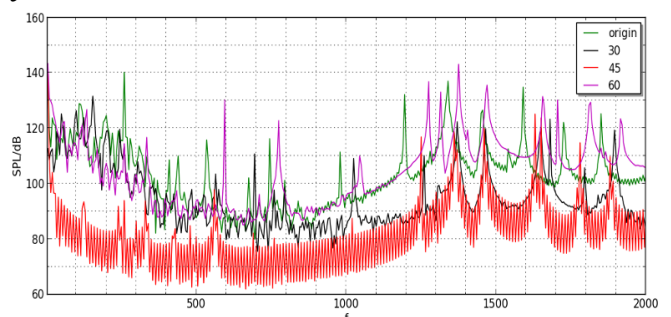

Fig. 6. The average sound pressure level of flow-induced noise from back wall chamfer with different angles.

It is found that the model with the back-wall chamfer at the angle of $30^{\circ}$ and $45^{\circ}$ can suppress the flow-induced noise. Moreover, the chamfer at the angle of $45^{\circ}$ can achieve effective suppression in the wide band range. However, the chamfer at the angle of $60^{\circ}$ increases the flow-induced noise.

Table 1. The average sound pressure level of flow-induced noise from back wall chamfer with different angles.

\begin{tabular}{|c|c|}
\hline angles & $\begin{array}{c}\text { Average sound pressure } \\
\text { level }\end{array}$ \\
\hline original model & $152.6 \mathrm{~dB}$ \\
\hline $30^{\circ}$ & $144.4 \mathrm{~dB}$ \\
\hline $45^{\circ}$ & $146.7 \mathrm{~dB}$ \\
\hline $60^{\circ}$ & $154.9 \mathrm{~dB}$ \\
\hline
\end{tabular}

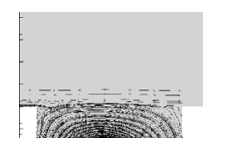

Origin

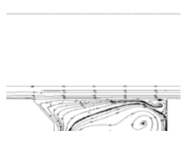

$30^{\circ}$

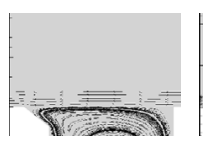

$45^{\circ}$

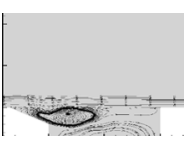

$60^{\circ}$
Fig. 7. The streamline diagram of back wall chamfer with different angles.

We had analyzed the mechanism that the back wall of the chamfer affects the flow-induced noise in the flow field. The chamfer at the angle of $30^{\circ}$ and $45^{\circ} \mathrm{can}$ influences the reattachment of the boundary layer at the trailing edge of the cavity, the fluctuation pressure at the trailing edge of the cavity is decreased. Then, the flow-induced noise is reduced. The chamfer at the angle of $60^{\circ}$ will generate eddies at the trailing edge of the cavity. The eddies are very unstable, which enhance the fluctuation pressure of the back wall of the cavity and increase average sound pressure level of flow-induced noise.

\subsection{Influence of flow-induce noise from back wall chamfer with airfoil surface}

Because of the back-wall chamfer at $45^{\circ}$ has better effect of noise reduction in wide frequency band; the chamfer is selected as the prototype. The plane chamfer is changed to the airfoil chamfer on the condition of the same angle. The NACA0410 airfoil surface, the NACA0420 airfoil surface, and the NACA0440 airfoil surface are used for simulation.

All of three airfoil surfaces reduce the average sound pressure level of flow-induced noise, and the effect of noise reduction is more obvious, when the frequency is above $1000 \mathrm{~Hz}$. Among them, the airfoil surface of NACA 0420 has the optimum effect. However, the airfoil surface reduces the effect of noise suppression if the frequency is below $500 \mathrm{~Hz}$.

Table 2. The average sound pressure level of flow-induce noise from back wall chamfer with different shapes.

\begin{tabular}{|c|c|}
\hline Back wall chamfer shape & $\begin{array}{c}\text { Average sound pressure } \\
\text { level }\end{array}$ \\
\hline plane & $146.7 \mathrm{~dB}$ \\
\hline NACA0410 & $137.0 \mathrm{~dB}$ \\
\hline NACA0420 & $128.1 \mathrm{~dB}$ \\
\hline NACA0440 & $131.0 \mathrm{~dB}$ \\
\hline
\end{tabular}




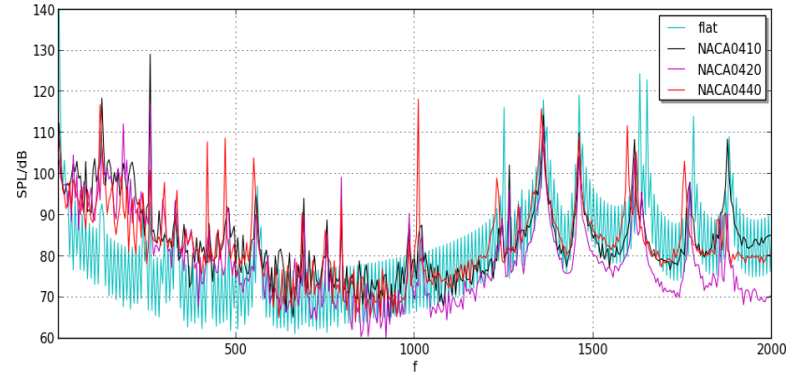

Fig. 8. The average sound pressure level of flow-induce noise from back wall chamfer with different shapes.

\section{Conclusions}

The simulation found that the back-wall chamfer can suppress the hydrodynamic noise of underwater three-dimensional cavity, and the effect of noise suppression is related to the angle. There is an optimal control angle, $45^{\circ}$. The airfoil chamfer has better effect of noise reduction than the straight chamfer. The suppression effect is mainly focused on the high frequency, which is related to the selected airfoil profile. The results of the study initially explored the effect of the angle of back wall chamfer on the suppression of hydrodynamic noise from the airfoil cavity at a fixed flow velocity.

It is believed that the results in the paper can provide some references for the noise reduction from the open cavities, especially in the submarines.

The work has been supported by the China Postdoctoral Science Foundation Funded Project, No.2017M611358 and also funded by National Key Research and Development Project, No.2016YFF0200906, Heilongjiang Province Foundation, No.GX17A015. Elsewhere, the work has been supported by the major project from Acoustic Science and Technology Laboratory, No.9140C200104140C20003, and also been funded the steady support plan from Acoustic Science and Technology Laboratory (Grant No.SSJSWDZC2018005). Furthermore, the work has been funded by the project from Key Laboratory of Acoustic Stealth (Grant No. 614220405011706), the Fundamental Research Funds for Central universities (Grant No.HEUCF180503), and funded by the China Scholarship Council, No.201706685061.

\section{References}

1. Rossiter J. E, Rep. Mem.3438, Aeronautical Research Council(1964)

2. Rockwell D, Naudascher E, Journal of fluid engineering, Transactions of the ASME, 100, 152165 (1978)

3. Seiichiro I, Hiroshi M, Osamu T, Ao-Kui X, Yu F, JSME International Journal, 49, 1092-1097 (2006)

4. Brenda H, Aeroacoustics, 9, 91-122 (2010)

5. N. Heeb, J. Kastner, E. Gutmarkand K. Kailasanath, Aeroacoustics, 12, 679-698 (2013)

6. WANG Y P, LI S, YANG X, Applied Acoustics, 111, 16-24 (2016)
7. YANG D G, FAN Z L, LI Ji Q, LUO X F, JIANG $\mathrm{W}$ M, Journal of Experiments in Fluid Mechanics, 24, 22-25 (2010)

8. Heatwole C M, Franchek M A, Bernhard R J, Herrick Laboratory Internal Report, 27, 7-97 (2005)

9. Heatwole C M, Franchek M A, Bernhard R J, Noise-Con 96, Seattle, Wash, 62-357 (1996) 